\section{Independent evidence backs call for a badger cull}

SIR - In your Editorial 'In for the cull' (Nature 450, 1; 2007), you implied that my assessment of the scientific evidence relating to bovine tuberculosis (TB) in cattle and badgers was influenced by political considerations. This attack on my integrity as the government's chief scientific adviser has no foundation whatsoever. The editorial staff of a journal surely understand fully the importance of scientific challenge and debate, as distinct from personal attacks. Yet your Editorial does not address the science.

As you point out, the scientific content of the report on cattle from the Independent Scientific Group (ISG), under the chairmanship of John Bourne, has been published in the scientific literature and hence peer-reviewed, but the conclusions reached by Bourne and his colleagues in the report have not.

My role is to provide independent scientific challenge and advice on important issues, such as this, in which the science is critical. To do my assessment, I assembled a team of independent, well-respected experts who brought international expertise in the necessary disciplines, particularly badger ecology, epidemiology, immunology and bovine TB. I had no idea what conclusions they would reach.

Although the scientific conclusions produced by my experts differ from the main conclusion of the ISG report, they nonetheless follow directly from the ISG's data, which clearly show that carrying out badger removal over a large area and a sustained period of time, together with cattle removal and other controls, would deliver an overall reduction in TB incidence in cattle herds. This is the only effective course of action until efficacious vaccines become available.

Bovine TB in cattle is the most serious endemic animal disease in the United Kingdom: the ISG reports a doubling of herd breakdown every 4.5 years in the high-incidence area. These data suggest that, in the randomized badger-culling trial, badgers could account for up to $40 \%$ of new confirmed incidents of bovine TB in cattle.

On the basis of the scientific evidence, I do not believe that we can control TB in cattle - and badgers - without removing the sources of infection in both species. Other countries have been unable to control TB in cattle without addressing the wildlife reservoir (N. E. Tweddle and P. Livingstone Vet. Microbiol. 40, 23-39; 1994).

I utterly reject any suggestion that I was - or could have been - influenced in my science advice by farmers, policy-makers or politicians. My mantra of openness, honesty and transparency continues unabated.

\section{David King}

Government Office for Science, Department of Universities, Innovation and Skills, Kingsgate House, 66-74 Victoria Street, London SW1E 6SW, UK

Nature has no reason to question Sir David's honesty, nor any evidence of political doctoring of his report. We stand by the concerns about the practice of scientific advice raised in the Editorial - Editor, Nature

\section{Kyoto: talks must include key aspects of science}

SIR - Gwyn Prins and Steve Rayner are correct to argue, in their Commentary 'Time to ditch Kyoto' (Nature 449, 973-975; 2007), that the Kyoto Protocol has failed and needs a radical rethink, but they do not diagnose the roots of this failure correctly. Lessons are less likely to be learned without this diagnosis.

There were two fundamental problems: one inherent in the negotiating model and the other in the issue structure that came to dominate the process. The first overestimated the possibility that science would counter the divergent interests of different states sufficiently for them to act in the greater global good; the second diminished the possibility that force of moral obligation would enhance this prospect.

A better understanding of the science might inform post-Kyoto negotiations more productively. For example, the logarithmic nature of carbon dioxide forcing, with each additional tonne having a smaller effect than the last, suggests that burdens should be less for countries industrializing now than for those that industrialized 100 years ago or more. And the 'Hansen alternative scenario' (J. Hansen et al. Proc. Natl Acad. Sci. USA 97, 9875-9880; 2000) suggests that much might be done by mitigating carbon-forcing agents, such as carbon soot from inefficient biofuel combustion, because this would bring enormous co-benefits from addressing indoor-air pollution in India and elsewhere.

'Minilateralism' in groups such as the G8+5 Climate Change Dialogue and the AsiaPacific Partnership on Clean Development and Climate (AP6), recently joined by Canada, is likely to be more productive than full multilateralism. But neither approach is going to be particularly productive unless key aspects of climate science inform the negotiations, and the normative discourse makes accurate and credible demands on parties such as the United States and Australia, which are unlikely to set aside their interests in the absence of such measures.

\section{Aynsley Kellow}

School of Government, University of Tasmania, Private Bag 22 Hobart 7001, Australia

\section{Kyoto: no time to rearrange deckchairs on the Titanic}

SIR - Gwyn Prins and Steve Rayner, in their Commentary (Nature 449, 973-975; 2007), manage to be perfectly right and utterly wrong at the same time. Their criticism of the bureaucratic Kyoto Protocol is justified on many crucial points (although they don't mention that the physical impact of the protocol on the climate system would be negligible even if it worked). The novelty of this summary of well-known deficiencies in the treaty is that the list comes from independent European scientists rather than White House mandarins. Is there anything substantially new beyond that provocation?

Yes, in the sense that Prins and Rayner boldly propagate a "bottom-up 'social learning" "approach to climate policy that aspires to "put public investment in energy R\&D on a wartime footing". I agree with the importance of both elements to twenty-first century climate protection, but doubt whether there is a solid causal chain linking them. Fine-scale measures and movements towards sustainability, as well as technological and institutional innovation strategies, are needed to decarbonize our industrial metabolism and to force policy-makers to face the challenges ahead. Fancy phrases such as "the silver buckshot" may help to sell the case.

Time is crucial, however. It is unlikely that a bottom-up, multi-option approach alone will be able to mobilize war-level climateprotection efforts by all the major emitters (including Russia, China and India) within the one or two decades left to avert an unmanageable planetary crisis. Without a 'global deal' - designed for effectiveness, efficiency and fairness and providing a framework to accommodate every nation there will be neither sufficient pressure nor appropriate orientation towards the climate solutions we desperately need. The bottom-up and top-down approaches are complementary and must be pursued interactively.

Kyoto is simply a miserable precursor of the global regime intended to deliver genuine climate stablization - and was never expected to be more. "Ditching" it now would render all the agonies involved completely meaningless after the event, denying the entire process of policy evolution the slightest chance to succeed. So, instead of rearranging the deckchairs on the Titanic through social learning, let us ditch pusillanimity.

\section{John Schellnhuber}

Potsdam Institute for Climate Impact Research, PO Box 601203, D-14412 Potsdam, Germany See Nature's climate blog at http://tinyurl.com/ 37g2pk. Readers are welcome to add their comments. 